\title{
Self-Driving Car
}

\author{
${ }^{1}$ Paras Dhawan, ${ }^{2}$ Manik Agarwal, ${ }^{3}$ Nikhil Kumar, ${ }^{4}$ Manish Singh Bisht, ${ }^{5}$ Prachi Agarwal \\ ${ }^{5}$ Assistant Professor, \\ ${ }^{12345}$ Department of CS \& IT, MIT, Moradabad, U.P., India
}

\begin{abstract}
The numbers of road accidents are increasing worldwide day by day and most of them are due to human errors including rash driving, carelessness and mishandling. Also the traffic is a big problem in the world. This problem can only be handled by driverless car. The motive is to develop a robo-car that drives without a driver on lanes. It should follow the lane lines on particular track and turn according to the lane. It should contain feature to turn left and right and must take U-turn after detecting end of lane. This can be done by constructing a set of hardware that enables image processing. Algorithms are applied on the fly i.e. image processing is done on real time data. The pollution caused by traffic is enormous and environment is affected adversely. The pollutants release by cars is very minute. The traffic and pollution affect us badly and waste our time and it is very inconvenient. Thus we need an cheap and convenient alternative in order to decrease this risk.
\end{abstract}

Index Terms: H-bridge, Inter-Integrated Circuit Communication, Master Slave Architecture, Perspective Transformation.

\section{INTRODUCTION}

This idea is to develop a hardware based embedded system in which master-slave architecture is used. We use Arduino UNO as a slave device while Raspberry Pi as a master device which give instructions to Arduino UNO. The slave device acts as a microcontroller which controls motor driver which in turn controls motors of robo car. Raspberry Pi is also used to implement various images processing task. The motor driver used is L-298 and 2 D.C gear motors with 1:50 gear ratio are used in this project. The camera is used to take input frames. Two power supply are used to give power to H-bridge and Raspberry Pi. For this purpose we use 9V D.C batteries.

\subsection{Levels of Autonomy}

Different cars are capable of different levels of self-driving, and are often described by researchers on a scale of 05.Autonomous vehicles are those cars that are driven by digital technologies with minimum or no human intervention. Thesecars are capable of driving and navigating them on the roads lanes by sensing the impact of environment. These cars are appeared as they are designed to occupy less space on road in order to get rid of traffic jams and reduce the probability of road accidents. But the progression is huge, in 2017, allowed automated vehicles on public roads are not completely autonomous, each one needs a human-driver who take cares when it is necessary to take back the control over the vehicle.

- Level 0: Each major system is controlled by humans

- Level 1: Very few systems, such as cruise control and automatic braking, are controlled by the vehicle (car), one at a time. Automation in level -1,some small steering or acceleration tasks are performed by car without intervention of human, but remaining things are fully under human control.

- Level 2: The car implements at least two automated functions simultaneously, like acceleration and steering, but humans are required for safe operation. Level-2 automation is similar to cruise control or original autopilot system on some Tesla vehicles, the car can automatically take safe and secure actions but the driver needs to be alert at the wheel.[1]

- Level 3: At this level, car will manage all safety and critical functions under certain circumstances, but the driver have to take over when alerted. Level-3 automation also requires a human driver, but the human is able to perform some safety-critical functions, over some traffic or environmental conditions. It have some 
potential dangers as humans pass the major tasks of driving to and from the car, that is why some car companies are interested to jump directly to level-4.

- Level 4: The car has full autonomy in some driving scenarios, although not to all. Autonomy in level-4 defines a car that drives itself almost all of time without any input from human, but may be programmed not to drive in unmapped areas or during bad weather situations. It is a car you can sleep in.

- Level 5: The car is completely able of self-driving in each and every situation. Level-5 automation means full automation in all circumstances.

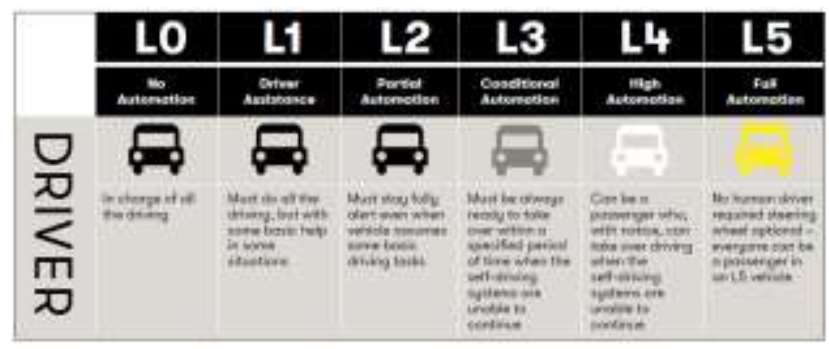

Fig.1 Autonomy Levels

\section{RELATED WORKS}

The Google team had been working on autonomous cars for many years and in recent years a working-prototype was shown by them. Apart from that, Google also supports other car manufacturers with driverless car technologies such as Toyota-Prius, Audi, and Lexus. Their own driverless vehicle uses Bosch sensors and other tools manufactured by LG and Sub-Continental companies. In 2014, a driverless car is planned by Google that would be available without pedal or wheels to make it available to the public by 2020, but according to the current situationits fulfillment is still less probable.

A landmark driverless car appeared in the 1980s, with Carnegie Mellon University's Navlab and ALV projects funded by United States' Defense Advanced Research Projects Agency-(DARPA) started in 1984 and MercedesBenz and Bundeswehr University Munich's EUREKA Prometheus Project in 1987. The ALV had also demonstrated self-driving speeds on two lane roads of 31 kilometer per hour i.e. $19 \mathrm{mph}$, with adding obstacle avoidance in 1986 and off road driving in day and night-time conditions by 1987. A huge milestone was achieved in 1995, with CMUNav-Lab 5 completing the first driverless coast to coast drive of the United States.

The Chief Executive Officer of Tesla, Elon Musk, claims that each car by Tesla will be completely autonomous within 2 years. The Tesla's S-model is a semi-self-propelled-car where various cars are able to learn from each other when working together. The sensor processed signals are sent to other cars thus these canalso develop each other. These information teacher cars exchange their information about changing lanes and detecting obstacles and these are continuously improving from day to day.

A group of graduates of the Massachusetts Institute of Technology (MIT) constructed the nuTonomy algorithm and its implementation especially for self-propelled cars. In Singapore, nuTonomy has already put sensors to the Mitsubishi i-MiEV electric car prototype. Thus nuTonomy algorithms can control the car on these complex and semi-urban roads through GPS and LiDAR sensors. Apart from that, in November 2016, they also announced that self-propelled cars will also be tested in Boston.

\section{PROBLEM DEFINITION}

The aim is to generate an H-Bridge that controls motors of the car and establishes a master-slave architecture to control H-bridge and processing the data which is captured through Camera. Therefore, the aim of this project is to 
develop a car that is able to provide a set of functions that make it safe, convenient and risk-free to use and built a set of hardware that act as a driverless car on a particular track.

\section{PROPOSED METHODOLOGY}

The various algorithms that can be applied on the image frames extracted include Gaussian Blur, Perspective Transform, Canny Edge Detection and Hough Transform. The purpose of using Gaussian Blur algorithm is to reduce noise and smoothen the frames extracted through camera. Basically, a Gaussian blur effect is created by convolving an image with a kernel of Gaussian values i.e. FIR kernel. Practically, the best way is to take advantage of the Gaussian blur separable property by dividing the process into two passes. During first pass basically a 1-D kernel is used to blur the image in only the hozizontal or vertical direction. The same one-dimensional kernel is used to blur in the remaining direction in the second pass. [2]

This has same effect as convolving with a two dimensional kernel in a single pass, but fewer calculations are required. Perspective Transformation also known as Bird's Eye View, in general terms, perspective means when human eyes see an object,it looks bigger when it is close to the eye and smaller when it is far from the eyes. Transformation means the transfer of an object from one state to another. Perspective transformation works in the same principle in which human vision and camera work that is the conversion of the $3 \mathrm{D}$ world into a $2 \mathrm{D}$ image.

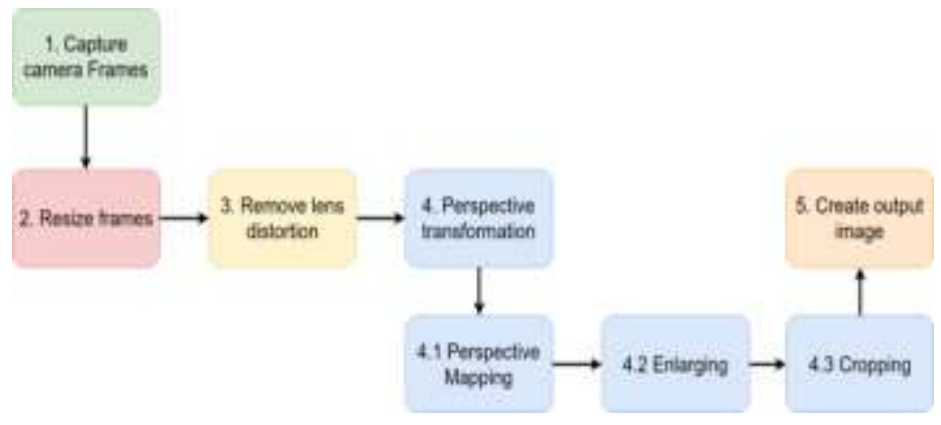

Fig. 2 Workflow of Perspective Transformation

\subsection{Workflow of Perspective Transformation}

Canny Edge Detection can be used to find lane lines in the frame. So it finds those set of points and point it out by white line. It defines a minimum threshold for intensity change and calculate gradient at every point in the frame and this process is continuously applied to all the frames. The steps are-

- Reduction in Noise

- $\quad$ Find Gradient of the Image

- Non-maximum Suppression

- Hysteresis and Thresholding

The Hough transformation is a feature-extraction technique used in analysis of image, computer vision, and DIP. The purpose of the technique is to find imperfect instances of objects within a certain class of shapes by a voting procedure. The algorithm uses the concept of hough space representation. It is used to for line detection. It defines a minimum cutoff to decide which lines are accepted or rejected. The following hardware is used in this project-

- Slave device- Arduino UNO

- $\quad$ Master device- Raspberry Pi 3B+ 
- Motor- D.C. gear motors (2 in quantity)

- Motor driver- L-298N

- Power Supply-9V D.C. Supply(2 in quantity)

- Camera- RaspiCam-V2

- CSI Cable

- Robo Car Chassis

- Micro SD Card-16 GB

\section{SIMULATED RESULTS ANDDISCUSSIONS}

In our project we have designed a hardware setup consists of two modules. First module is Lane Detection and Tracking and second is Lane End Detection and U-turn implementation. Basically we are trying to design a robo-car setup which is capable of finding and tracking lane lines and turn accordingly.

This will work for both straight and curved paths but on a particular track. In second module, we implement functions like finding lane lines, U-turn implementation and obstruction avoidance by turning left or right.

The backbone of our project is Inter-Integrated Circuit Communication (I2C) that allows Arduino and Raspberry Pi to act as a Master-Slave devices. We have detected lane lines on both side of the car on our track using opencv library.

Also we implement functions like Forward, Backward, Left and Right on Straight lanes. We also implement tracking of lanes on both straight and curved tracks.

The robo car follows the curve of the lane without giving external commands. For this we use Canny Edge Detection algorithm, Perspective view, Gaussian Blur and Hough Space Conversion.. All processing of data will be done by Raspberry Pi and Arduino controls the motor driver which controls the motors.

For the implementation of Lane Tracking we have built a track on which the car move and follow the lane lines. Also the master-slave architecture of arduino and raspberry pi is designed in this module. The camera captures images and processing is done frame by frame on the fly.

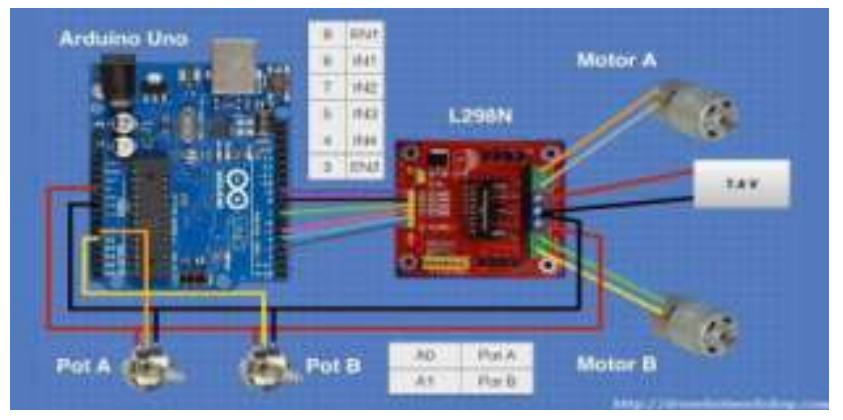

Fig. 3 Connections of H-Bridge

We have implemented functions like lane end detection, U-turn implementation and obstruction avoidance. For lane end detection, the robo car following the curve on seeing the end of lane stops. This can be done by combined setup. But there is a lag in between because of real time data being processed.

U-turn implementation is done on the particular track comprises of two lane road. When one lane ends car stops 
and takes U-turn. For the implementation of object avoidance, we use an ultrasonic or infrared sensor that detects distance from object and either stop or turns left or right.

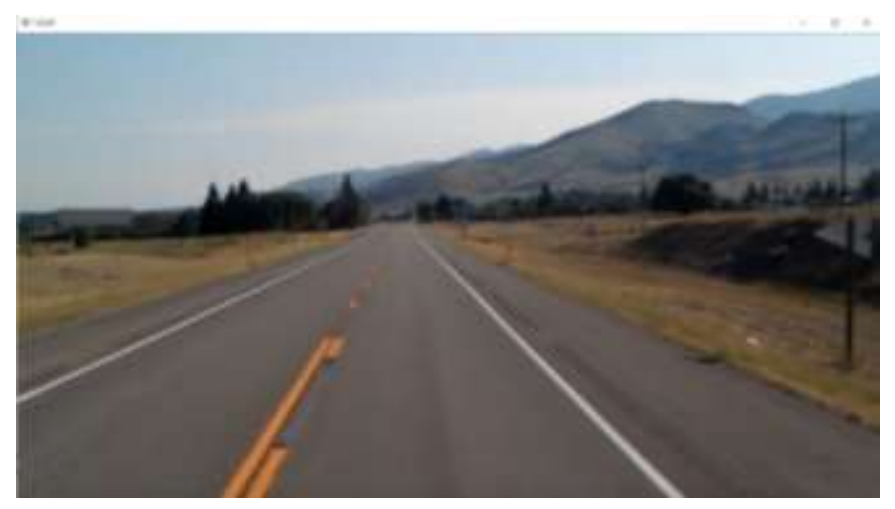

Fig. 4:input image

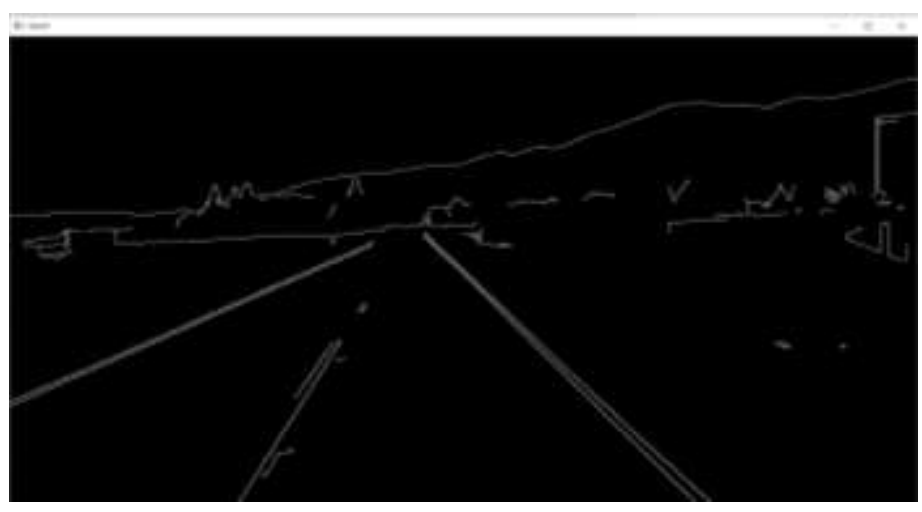

Fig. 5 After applying Canny Edge Detection

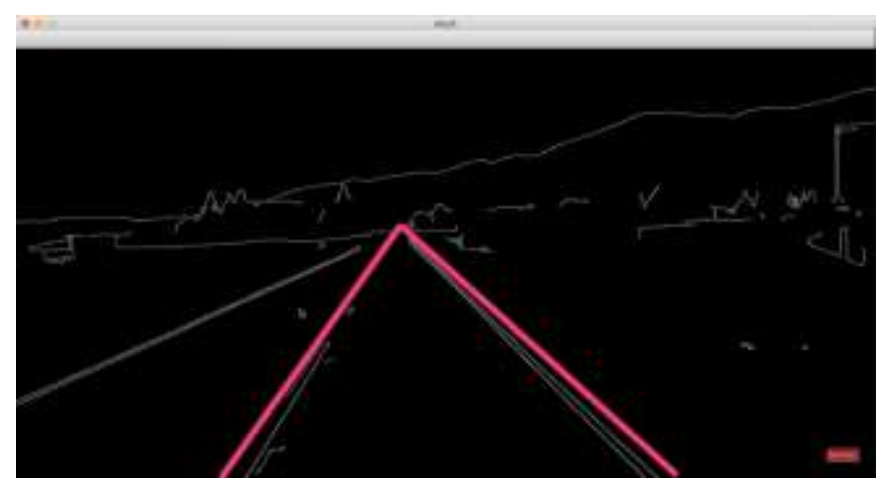

Fig. 6 Finding Area of Interest. 


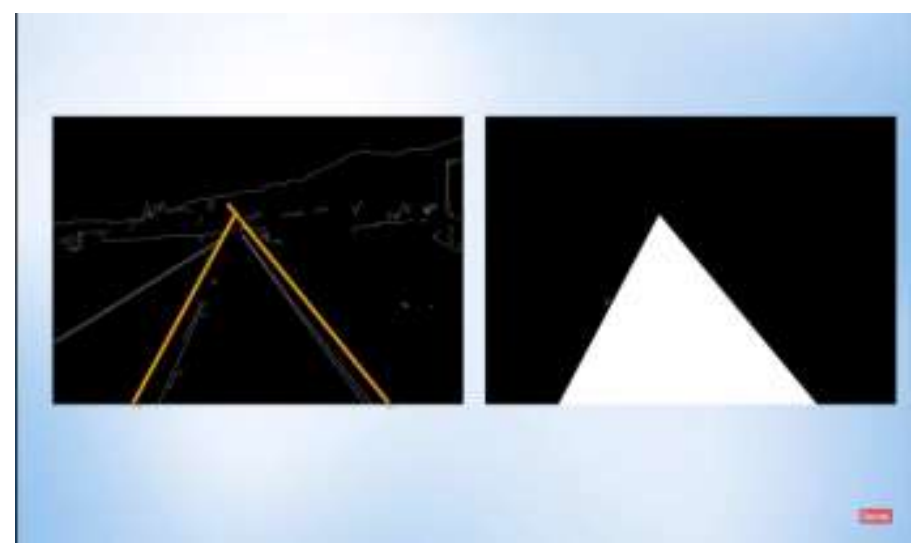

Fig. 7:Finding mask

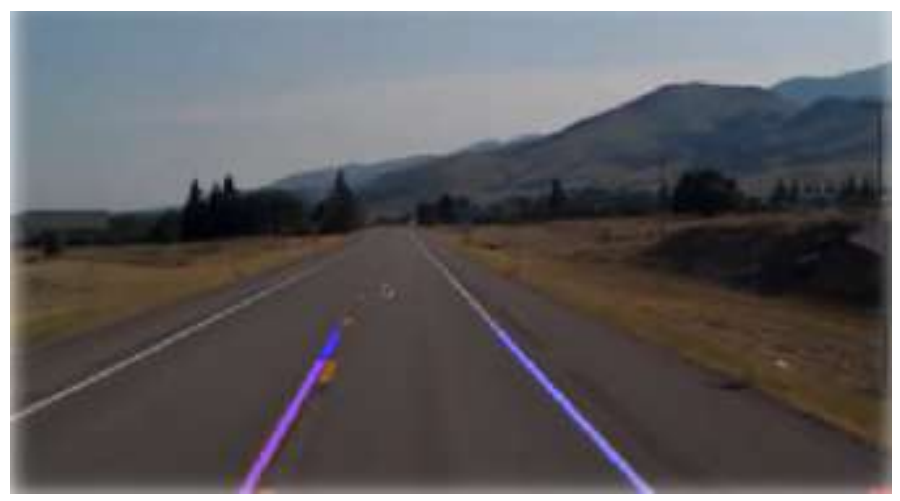

Fig. 8: Desired Output

\section{CONCLUSION}

Autonomous Vehicles (AV) has been an active area of research for recent decades but majorly in the last five years. Recently, the joint efforts of universities, researchers and manufacturers have brought AVs to almost readiness. These have very lower transportation cost. In one such estimate, autonomous vehicles impacts in terms of crash savings, reduction in travel time, fuel efficiency, and parking-benefits may be as much as 2000 dollars per annum per AV and may be as high as 4000 dollars when comprehensive crash costs are accounted. These are still in the infancy stage. There is a long road to travel before achieving maturity, implementation, and market-release.[5]

As far as concerned about this project we can include various additional features in future like-

- We can add module that to train algorithm to detect stop symbol.

- We can add module to detect traffic lights and act accordingly.

- We can use deep learning algorithms to increase performance but it need enormous processing power and storage.

- We can use LIDAR or RADAR techniques for detecting obstruction in the path and this will make it smooth but costlier. 


\section{FUTURE DIRECTIONS}

There are still several problems in the path and we have to face several implementation challenges. Environmentalperception remains the most important challenge to reliable, smooth and securedriving. There is a wide rangeof research questions covering this wide area that will need to be addressed and answered including but not constrainedto customer acceptance, impacts on society, communication technologies, ethical issues, planning and implementing, standards, and policy issues. Software challenges such as security and integrity of system have also been emerged as major issues to be addressed. Thus we have a number of policy implications including the problems for policymakers to streamline and regulate many vehicles with different operating circumstances. It is also important for policymakers to ensure that drivers understand these vehicle capabilities and can operate them with utmost safety. [3]

\section{REFERENCES}

[1] A. Rassõlkin, L. Gevorkov, T. Vaimann, A. Kallaste and R. Sell(2018), "Calculation of the traction effort of ISEAUTO self-driving vehicle", 25th International Workshop on Electric Drives: Optimization in Control of Electric Drives (IWED), pp. 1-5.

[2] Berger, C. and Dukaczewski, M. (2014), "Comparison of architectural design decisions for resource-constrained self-driving cars - a multiple case-study", Proceedings of the Informatik, pp. 2157-2168.

[3] Computerworld (2012), "Self-driving cars a reality for 'ordinary people' within 5 years, says Google's Sergey Brin”, Communications of the ACM,

[4] Conejero, J.A., Jord, N.C. and Sanabria-Codesal, E. (2016), "An algorithm for self-organization of driverless vehicles of a car-rental service", Nonlinear Dynamics, Vol. 84 No. 1, pp. 107-114.

[5] E. Ackerman(2016), "Lidar that will make self-driving cars affordable [News]", IEEE Spectrum, vol. 